EUROPEAN JOURNAL OF PURE AND APPLIED MATHEMATICS

Vol. 15, No. 1, 2022, 290-313

ISSN 1307-5543 - ejpam.com

Published by New York Business Global

\title{
Solving fuzzy system of Volterra integro-differential equations by using Adomian decomposition method
}

\author{
Mahasin Thabet Younis ${ }^{1}$, Waleed Al-Hayani ${ }^{1, *}$ \\ ${ }^{1}$ Department of Mathematics, College of Computer Science and Mathematics, \\ University of Mosul, Iraq
}

\begin{abstract}
In this paper, the Adomian decomposition method and Modified Technique are successfully applied to find the approximate solutions of the fuzzy system of Volterra integro-differential equations. The approximate solutions obtained have been improved by using the iteration of the integral equation and the numerical solution with the Simpson rule and Trapezoidal rule. These proposed methods gave excellent results close to the exact solution. The results show that the present method is very straightforward and effective.
\end{abstract}

2020 Mathematics Subject Classifications: 03E72, 26A42, 45J05

Key Words and Phrases: Fyzzy numbers, Fuzzy Systems of Volterra integro-differential equations, Adomian decomposition method, Modified technique, Adomian polynomials

\section{Introduction}

Integral equations are used in variety scientific and technical fields, especially in engineering fields, where they were considered from a period not briefly one of the most important tools in applied mathematics. Mathematical modeling problems common in the real world and in our lives are the result of analysis of differential equations, integral equations, integro-differential equations and statistical equations, and others.

Many mathematical formulations of some physical phenomena contain, integro-differential equations, these equations appear in fluid mechanics, biological models, kinetic physics and chemical reactions, as well as, integro-differential equations appear in many physical processes. Like glass formation and nano-dynamics [1]. There are many effective ways to find approximate solutions and numerical and analytical solutions to linear and nonlinear problems of integral equations and integro-differential equations used over decades Volterra integro-differential equations which has continuous kernel [1]. Approximation solution based on basis functions has been utilized to estimate solutions of integral equation in recent years.

${ }^{*}$ Corresponding author.

DOI: https://doi.org/10.29020/nybg.ejpam.v15i1.

Email addresses: mahasin_thabet@uomosul.edu.iq (M. T. Younis), waleedalhayani@uomosul.edu.iq, waleedalhayani@yahoo.es (W. Al-Hayani) 
Basic concept of fuzzy was first introduced by Professor Zadeh in 1965 after his publications on fuzzy set theory $[2,3]$. In the beginning of the 1980s, Adomian [4-6] proposed a new and fruitful method (so-called decomposition method) for solving linear and nonlinear (algebraic, differential, partial differential, integral, etc.) equations. It has been shown that this method yields a rapid convergence of the solutions series to linear and nonlinear deterministic and stochastic equations.

Many recent years solving fuzzy integro-differential equations, fuzzy Volterra-Fredholm integral equation and fuzzy Volterra-Fredholm integro-differential equations require appropriate and applicable definitions of fuzzy function [7-10], the fuzzy systems of integral equations have attracted increasing attention, with regard to fuzzy control, have been developed mathematical models used in many problems of physics, biology, chemistry, engineering, and in other fields depend on an integral equation [11].

$\mathrm{ADM}$ is an analytical technique that uses Adomian polynomials to evaluate the approximate solutions. This method does not simplify or discretize the problem, and it can be used to solve both linear and non-linear problems [12].

The ADM has been used to solve Volterra integral equations, integro-differential equations and Fredholm integro-differential equations of various types in the literature [13, 14]. Dalal Adnan and Eman Ahmed [15] have been examined the topic of population expansion. Arikglu and Ozkol [12] have been applied Differential Transform Method (DTM) on both integral equation and integro-differential equation system. Hassan and Peter [16] solve new iterative method with a reliable algorithm and applied to the systems of Volterra integro-differential equations. Berenguer et al [17] have been solved systems of integrodifferential equations using numerical of fixed Point. Biazar and Aminikhah [18] have been used Variational iteration method (VIM) for solving nonlinear integral-differential equations.

Bani Issaa et al [19] applied numerical methods to slove the fuzzy integro-differential equations of the second kind. Shabestari et al [20] have been solved Fuzzy Volterra Integrodifferential equations of fractional order by Bernoulli Wavelet method. Das and Talukdar [21] solved fuzzy integro-differential equation by using fuzzy Laplace Transformation. Mikaeilvand et al [22] applied the differential transform method (DTM) to solve fuzzy integro-differential equation.

The main objective of this article is to use the Standard Adomian Decomposition Method (ADM) and Modified Technique (MT) to solve the fuzzy systems of linear and non-linear four Volterra integro-differential equations (VIDEs) with a comparison between the both techniques and improved the approximate solutions obtained for the system of non-linear four VIDEs by using the iteration of the integral equation and the numerical solution with the Simpson rule and Trapezoidal rule.

\section{Basic Concepts}

Fuzzy numbers are generalized classical real numbers, and we can define them as a fuzzy subset of the real line with some additional features. The concept of a fuzzy number is essential for fuzzy analysis, fuzzy integral equations, as well as a useful tool in a variety 
of fuzzy set applications. The basic definitions of fuzzy numbers are the following:

Definition 1. (Fuzzy set) [23]: A set $\tilde{A}=\left\{\left(t, M_{\tilde{A}(t)}\right), t \in X\right\}$ is called a fuzzy set where $M_{\tilde{A}(t)}$ is the membership function of fuzzy set $A$ is defined by $M_{\tilde{A}(t)}: X \rightarrow[0,1]$, and the value of $M_{\tilde{A}(t)}$ is called the membership degree $X$.

Definition 2. (Fuzzy number) [24, 25]: A fuzzy number is a map $\tilde{u}: R \rightarrow[a, b]$, which satisfying

(i) $\tilde{u}$ is upper semi-continuous function.

(ii) $\tilde{u}(t)=0$ outside some interval $[a, d]$.

(iii) There are real numbers $b, c$ such $a \leq b \leq c \leq d$

i) $\tilde{u}(t)$ is a monotonic increasing function on $[a, b]$.

ii) $\tilde{u}(t)$ is a monotonic decreasing function on $[c, d]$.

iii) $\tilde{u}(t)=1$ for all $t \in[b, c]$.

Definition 3. [25-27]: A fuzzy number $\tilde{u}$ in a parametric form is a pair $(\underline{u}, \bar{u})$ of a function $\underline{u}(r), \bar{u}(r), 0 \leq r \leq 1$; which satisfy the requirements

(i) $\underline{u}(r)$ is a left continuous function and bounded monotonic increasing.

(ii) $\bar{u}(r)$ is a left continuous function and bounded monotonic decreasing.

(iii) $\underline{u}(r) \leq \bar{u}(r), 0 \leq r \leq 1$

The triangular fuzzy number $[2,20,28]$, which is defined as a fuzzy set in $\mathbb{R}$ and characterized by an ordered triple $u=(a, b, c) \in \mathbb{R}^{3}$ with $a \leq b \leq c$ such that $\underline{u}(r)=$ $a+(b-a) r$ and $\bar{u}(r)=c-(c-b) r$, are the endpoints of $r$-level sets for all $r \in[0,1]$ is a popular fuzzy number:

We can represent a crisp number $x$ by $(\underline{u}(r), \bar{u}(r))=(x, x), 0 \leq r \leq 1$. By appropriate definitions, the fuzzy number space $\{\underline{u}(r) \leq \bar{u}(r)\}$ becomes a convex cone $E^{1}$ which could be isometrically and isomorphically into a Banach space [29]. Let $\tilde{x}=(\underline{x}(r), \bar{x}(r))$, $\tilde{y}=(\underline{y}(r), \bar{y}(r)), 0 \leq r \leq 1$, and $k \in \mathbb{R}$. Then

(i) $\tilde{x}=\tilde{y}$ iff $\underline{x}(r)=\underline{y}(r), \bar{x}(r)=\bar{y}(r)$.

(ii) $\tilde{x}+\tilde{y}=(\underline{x}(r)+\underline{y}(r), \bar{x}(r)+\bar{y}(r))$.

(iii) $\tilde{x}-\tilde{y}=(\underline{x}(r)-\bar{y}(r), \bar{x}(r)-\underline{y}(r))$.

(iv) $k \tilde{x}= \begin{cases}(k \underline{x}, k \bar{x}), & k \geq 0 \\ (k \bar{x}, k \underline{x}), & k<0\end{cases}$ 
Definition 4. ( $r$-level) $[12,25,27,30]$ : Let $E$ be the set of all fuzzy number on $\mathbb{R}$, we denoted $[u]^{r} r$-level set of a fuzzy number $u \in E, 0 \leq r \leq 1$, it is a mapping between close interval $[0,1]$ to power set of $\mathbb{R}$, where

$$
[u]^{r}= \begin{cases}{[a(r), b(r)],} & r \in[0,1] \\ c l(\operatorname{supp}(u)), & r=0\end{cases}
$$

$[u]^{r}$ is closed and bounded interval $[\underline{u}(r), \bar{u}(r)]$ where $\underline{u}(r)$ denotes the left-hand endpoint of $[u]^{r}$ and $\bar{u}(r)$ denotes the right-hand endpoint of $[u]^{r}$ since each $u \in \mathbb{R}$ can be observed as defined by $\tilde{u}=\left\{\begin{array}{lll}1 & \text { if } t=u \\ 0 & \text { if } t \neq u^{\prime}\end{array}\right.$

where $c l(\operatorname{supp}(u))=$ closure of support $u$ and $\sup p(u)=\{t: u(t)>0\}$.

Definition 5. [23]: Let $u=(\underline{u}(r), \bar{u}(r)), v=(\underline{v}(r), \bar{v}(r)), 0 \leq r \leq 1$ be two any arbitrary fuzzy numbers and $k$ is scalar, we define the operation of a fuzzy number

(i) $\underline{(u+v)}(r)=(\underline{u}(r)+\underline{v}(r)), \overline{(u+v)}(r)=(\bar{u}(r)+\bar{v}(r))$.

(ii) $(u-v)(r)=(\underline{u}(r)-\bar{v}(r)), \overline{(u-v)}(r)=(\bar{u}(r)-\underline{v}(r))$.

(iii) $k \breve{u}= \begin{cases}(k \underline{u}(r), k \bar{u}(r)), & k \geq 0 \\ (k \bar{u}(r), k \underline{u}(r)), & k<0\end{cases}$

(iv) $\tilde{u} . \tilde{v}=\left\{\begin{array}{l}\underline{u v}(r)=\max \{\underline{u}(r) \underline{v}(r), \underline{u}(r) \bar{v}(r), \bar{u}(r) \underline{v}(r), \bar{u}(r) \bar{v}(r)\} \\ \overline{u v}(r)=\min \{\underline{u}(r) \underline{v}(r), \underline{u}(r) \bar{v}(r), \bar{u}(r) \underline{v}(r), \bar{u}(r) \bar{v}(r)\}\end{array}\right.$

Definition 6. (The fuzzy Riemann integral) [31]: We say that

1- $\widetilde{f}(t)$ is a fuzzy valued function if $\widetilde{f}: X \rightarrow F$.

2- $\widetilde{f}(t)$ is a closed fuzzy valued function if $\widetilde{f}: X \rightarrow F_{c l}$.

3- $\widetilde{f}(t)$ is a bounded fuzzy valued function if $\widetilde{f}: X \rightarrow F_{b}$.

We denote $A_{r}=\left[\int_{a}^{b} \widetilde{f}_{r}^{L}(s) d s, \int_{a}^{b} \widetilde{f}_{r}^{U}(s) d s\right]$. If $\tilde{f}(t)$ is a fuzzy valued function on $[a, b]$ that is closed and bounded, then the fuzzy Riemann integral $\int_{a}^{b} \widetilde{f}(s) d s$, is a closed fuzzy number. Furthermore, the $[u]^{r}$ set of $\int_{a}^{b} \tilde{f}(s) d s$ is

$$
\left(\int_{a}^{b} \widetilde{f}(s) d s\right)_{r}=\left[\int_{a}^{b} \widetilde{f}_{r}^{L}(s) d s, \int_{a}^{b} \widetilde{f}_{r}^{U}(s) d s\right] .
$$

\section{Fuzzy System of Volterra Integro-Differential Equations}

When a physical system is modeled in a differential sense, the result is a fuzzy integral equation or a fuzzy integro-differential equation, and hence the solution of fuzzy integro-differential equations is important in science and engineering. Analytically, nonlinear fuzzy integro-differential equations are difficult to solve, and accurate solutions are rare [10]. 
We consider the following fuzzy system of Volterra integro-differential equations (FSVIDEs) of the second kind [17, 18]:

$$
\widetilde{u_{i}^{\prime \prime}}(x, \alpha)=\widetilde{f}_{i}(x, \alpha)+\sum_{j=1}^{2} \lambda_{i j} \int_{0}^{x} K_{i j}(x, t) \widetilde{F_{i j}}(\widetilde{u}(t, \alpha)) d t, \quad i=1,2
$$

with the initial conditions

$$
\widetilde{u_{i}}(0, \alpha)=\left(\underline{a_{i}}(\alpha), \overline{a_{i}}(\alpha)\right), \quad \widetilde{u_{i}^{\prime}}(0, \alpha)=\left(\underline{b_{i}}(\alpha), \overline{b_{i}}(\alpha)\right), \quad i=1,2
$$

where $\lambda_{i j} \neq 0, i, j=1,2$ are real constants, $\widetilde{u}(t, \alpha)=\left(\widetilde{u_{1}}(t, \alpha), \widetilde{u_{2}}(t, \alpha)\right)^{T}, 0 \leq t \leq x$, $a \leq x \leq b, \widetilde{u}_{i}(x, \alpha)$ are unknown functions, $\widetilde{f}_{i}(x, \alpha)$ and the kernels $K_{i j}(x, t)$ are analytical functions, $\widetilde{F_{i j}}(\widetilde{u}(t, \alpha))$ are linear or non-linear functional of the unknown functions $\widetilde{u}_{i}(x, \alpha)$. Under the appropriate conditions $\widetilde{f}_{i}(x, \alpha)$ and $K_{i j}(x, t)$, the FSVIDEs $(1)$ have a unique continuous solution $\widetilde{u_{i}}(x, \alpha)$ on the given interval $[a, b]$.

The parametric form of the given FSVIDEs (1) can be written as

$$
\left\{\begin{array}{l}
\underline{u_{i}^{\prime \prime}}(x, \alpha)=\underline{f_{i}}(x, \alpha)+\sum_{j=1}^{2} \lambda_{i j} \int_{0}^{x} K_{i j}(x, t) \underline{F_{i j}}(\underline{u}(t, \alpha)) d t \\
\overline{u_{i}^{\prime \prime}}(x, \alpha)=\overline{f_{i}}(x, \alpha)+\sum_{j=1}^{2} \lambda_{i j} \int_{0}^{x} K_{i j}(x, t) \overline{F_{i j}}(\bar{u}(t, \alpha)) d t
\end{array} i=1,2\right.
$$

\section{Adomian Decomposition Method}

The Adomian decomposition method gives the solution of the FSVIDEs (1) as an infinite series usually converging to the closed form solution. To solve the FSVIDEs (1) and (2) by the Adomian's technique, assume an infinite series solution for the unknowns functions $\widetilde{u_{i}}(x, \alpha)=\left[\underline{u_{i}}(x, \alpha), \overline{u_{i}}(x, \alpha)\right], i=1,2$ given by $[19,21,23]$ as follows

$$
\begin{aligned}
& \underline{u_{i}}(x, \alpha)=\sum_{n=0}^{\infty} \frac{u_{i, n}}{(x, \alpha),} \quad i=1,2 \\
& \overline{u_{i}}(x, \alpha)=\sum_{n=0}^{\infty} \overline{u_{i, n}}(x, \alpha),
\end{aligned}
$$

and decomposing non-linear functions $\widetilde{F_{i j}}(\widetilde{u}(t, \alpha))=\left[\underline{F_{i j}}(\underline{u}(t, \alpha)), \overline{F_{i j}}(\bar{u}(t, \alpha))\right], i, j=$ 1,2 as

$$
\begin{aligned}
& \underline{F_{i j}}(\underline{u}(t, \alpha))=\sum_{n=0}^{\infty} \frac{A_{i j, n},}{\overline{F_{i j}}}(\bar{u}(t, \alpha))=\sum_{n=0}^{\infty} \overline{A_{i j, n}},
\end{aligned}
$$


where $\widetilde{A_{i j, n}}=\left[\underline{A_{i j, n}}, \overline{A_{i j, n}}\right], n \geq 0$, are polynomials (so called Adomian polynomials) [3-6] of $\widetilde{u_{i, 0}}(t, \alpha), \widetilde{u_{i, 1}}(t, \alpha), \ldots, \widetilde{u_{i, n}}(t, \alpha), i=1,2$ given by

$$
\begin{array}{ll}
\underline{A_{i j, n}}=\frac{1}{n !} \frac{\partial^{n}}{\partial \lambda^{n}}\left[F\left(\sum_{k=0}^{\infty} \lambda^{i} \underline{u_{i, k}}(t, \alpha)\right)\right]_{\lambda=0}, \quad i, j=1,2 \\
\overline{A_{i j, n}}=\frac{1}{n !} \frac{\partial^{n}}{\partial \lambda^{n}}\left[F\left(\sum_{k=0}^{\infty} \lambda^{i} \overline{u_{i, k}}(t, \alpha)\right)\right]_{\lambda=0}, & n=0,1,2, \ldots
\end{array}
$$

Applying the Adomian's technique as in [4-6], the FSVIDEs (3) can be written as

$$
\left\{\begin{array}{l}
\underline{L u_{i}}(x, \alpha)=\underline{f_{i}}(x, \alpha)+\sum_{j=1}^{2} \lambda_{i j} \int_{0}^{x} K_{i j}(x, t) \underline{N_{i j}} d t \\
\overline{L u_{i}}(x, \alpha)=\overline{f_{i}}(x, \alpha)+\sum_{j=1}^{2} \lambda_{i j} \int_{0}^{x} K_{i j}(x, t) \overline{N_{i j}} d t
\end{array}\right.
$$

where $\underline{L u_{i}}(x, \alpha)=\frac{d^{2} \underline{u}_{i}}{d x^{2}}, \overline{L u_{i}}(x, \alpha)=\frac{d^{2} \overline{u_{i}}}{d x^{2}}, i=1,2$ are the linear operators and $\underline{N_{i j}}=$ $F_{i j}(\underline{u}(t, \alpha)), \overline{N_{i j}}=\overline{F_{i j}}(\bar{u}(t, \alpha)), i, j=1,2$ are the non-linear operators. Operating on both sides of the FSVIDEs (7) with the inverse operator of $L$ (namely $L^{-1}=\int_{0}^{x} \int_{0}^{x}[\cdot] d t d t$ ) yields

$$
\left\{\begin{array}{l}
\underline{u_{i}}(x, \alpha)=\underline{u_{i}}(0, \alpha)+\underline{u_{i}^{\prime}}(0, \alpha) x+L^{-1} \underline{f_{i}}(x, \alpha)+L^{-1}\left[\sum_{j=1}^{2} \lambda_{i j} \int_{0}^{x} K_{i j}(x, t) \underline{N_{i j}} d t\right] \\
\overline{u_{i}}(x, \alpha)=\overline{u_{i}}(0, \alpha)+\overline{u_{i}^{\prime}}(0, \alpha) x+L^{-1} \overline{f_{i}}(x, \alpha)+L^{-1}\left[\sum_{j=1}^{2} \lambda_{i j} \int_{0}^{x} K_{i j}(x, t) \overline{N_{i j}} d t\right]
\end{array} i=1,2\right.
$$

Using (4) and (5) into the FSVIDEs (8) it follows that

$$
\left\{\begin{aligned}
\sum_{n=0}^{\infty} \underline{u_{i, n}}(x, \alpha) & =\underline{u_{i}}(0, \alpha)+\underline{u_{i}^{\prime}}(0, \alpha) x+L^{-1} \underline{f_{i}}(x, \alpha) \\
+ & L^{-1}\left[\sum_{j=1}^{2} \lambda_{i j} \int_{0}^{x} K_{i j}(x, t) \sum_{n=0}^{\infty} \underline{A_{i j, n}} d t\right], \quad i=1,2 \\
\sum_{n=0}^{\infty} \overline{u_{i, n}}(x, \alpha) & =\overline{u_{i}}(0, \alpha)+\overline{u_{i}^{\prime}}(0, \alpha) x+L^{-1} \overline{f_{i}}(x, \alpha) \\
+ & L^{-1}\left[\sum_{j=1}^{2} \lambda_{i j} \int_{0}^{x} K_{i j}(x, t) \sum_{n=0}^{\infty} \overline{A_{i j, n}} d t\right]
\end{aligned}\right.
$$

Using the fuzzy Standard ADM, according to (9), the lower iterations (L) are then determined in the following recursive way:

$$
\begin{cases}\underline{u_{i, 0}}(x, \alpha)=\underline{u_{i}}(0, \alpha)+\underline{u_{i}^{\prime}}(0, \alpha) x+L^{-1} \underline{f_{i}}(x, \alpha), & \\ \underline{u_{i, n+1}}(x, \alpha)=L^{-1}\left[\sum_{j=1}^{2} \lambda_{i j} \int_{0}^{x} K_{i j}(x, t) \underline{A_{i j, n}} d t\right], & n=0,1,2, \ldots\end{cases}
$$


and the upper iterations (U) are

$$
\begin{cases}\overline{u_{i, 0}}(x, \alpha)=\overline{u_{i}}(0, \alpha)+\overline{u_{i}^{\prime}}(0, \alpha) x+L^{-1} \overline{f_{i}}(x, \alpha), & i=1,2 \\ \overline{u_{i, n+1}}(x, \alpha)=L^{-1}\left[\sum_{j=1}^{2} \lambda_{i j} \int_{0}^{x} K_{i j}(x, t) \overline{A_{i j, n}} d t\right], & n=0,1,2, \ldots\end{cases}
$$

Using the fuzzy Modified Technique (MT) [11], according to (9), Lower iterations (L) are then calculated in a recursive manner as follows:

$$
\left\{\begin{array}{l}
\underline{u_{i, 0}}(x, \alpha)=\underline{u_{i}}(0, \alpha)+\underline{u_{i}^{\prime}}(0, \alpha) x \\
\underline{u_{i, 1}}(x, \alpha)=L^{-1} \underline{f_{i}}(x, \alpha)+L^{-1}\left[\sum_{j=1}^{2} \lambda_{i j} \int_{0}^{x} K_{i j}(x, t) \underline{A_{i j, 0}} d t\right] \\
\begin{array}{l}
i=1,2 \\
n=1,2, \ldots
\end{array} \\
\underline{u_{i, n+1}}(x, \alpha)=L^{-1}\left[\sum_{j=1}^{2} \lambda_{i j} \int_{0}^{x} K_{i j}(x, t) \underline{A_{i j, n}} d t\right]
\end{array}\right.
$$

and the upper iterations (U) are

$$
\left\{\begin{array}{l}
\overline{u_{i, 0}}(x, \alpha)=\overline{u_{i}}(0, \alpha)+\overline{u_{i}^{\prime}}(0, \alpha) x \\
\overline{u_{i, 1}}(x, \alpha)=L^{-1} \overline{f_{i}}(x, \alpha)+L^{-1}\left[\sum_{j=1}^{2} \lambda_{i j} \int_{0}^{x} K_{i j}(x, t) \overline{A_{i j, 0}} d t\right] \\
\overline{i=1,2} \\
\overline{u_{i, n+1}}(x, \alpha)=L^{-1}\left[\sum_{j=1}^{2} \lambda_{i j} \int_{0}^{x} K_{i j}(x, t) \overline{A_{i j, n}} d t\right]
\end{array}\right.
$$

Thus, all components $\left(\widetilde{u_{i, n}}(x, \alpha)=\left[\underline{u_{i, n}}(x, \alpha), \overline{u_{i, n}}(x, \alpha)\right], i=1,2\right)$ of $\widetilde{u_{i}}(x, \alpha)=$ $\left[\underline{u_{i}}(x, \alpha), \overline{u_{i}}(x, \alpha)\right], i=1,2$ can be calculated once the $\widetilde{A_{i j, n}}$ are given for $i, j=1,2, \ldots, m$ and $n=0,1,2, \ldots$. We then define the $n$-term approximants to the solutions $\widetilde{u}_{i}(x, \alpha)=$ $\left[\underline{u_{i}}(x, \alpha), \overline{u_{i}}(x, \alpha)\right], i=1,2$ by $\phi_{i, n}\left[\underline{u_{i}}(x, \alpha)\right]=\sum_{k=0}^{n-1} \underline{u_{i, k}}(x, \alpha), i=1,2$ with $\lim _{n \rightarrow \infty} \phi_{i, n}\left[\underline{u}_{i}(x, \alpha)\right]=$ $\underline{u_{i}}(x, \alpha), i=1,2$ and by $\phi_{i, n}\left[\overline{u_{i}}(x, \alpha)\right]=\sum_{k=0}^{n-1} \overline{u_{i, k}}(x, \alpha), i=1,2$ with $\lim _{n \rightarrow \infty} \phi_{i, n}\left[\overline{u_{i}}(x, \alpha)\right]=$ $\overline{u_{i}}(x, \alpha), i=1,2$.

\section{Applications and numerical results}

The fuzzy systems of linear and non-linear Volterra integro-differential equations are displayed in the following two problems, and we use ADM and MT to achieve approximate exact solutions. In the problem 2. The maximum errors are defined as follows to demonstrate the great accuracy of the solution outcomes when compared to the exact solutions:

$$
L_{\infty}[a, b]=\left\|u_{\text {Exact }}\left(x_{i}, \alpha\right)-\phi_{i, n}\left(x_{i}, \alpha\right)\right\|_{\infty},
$$




$$
\begin{aligned}
L_{2, S}[a, b] & =\sqrt{\sum_{i=0}^{n}\left[u_{\text {Exact }}\left(x_{i}, \alpha\right)-\phi_{i, n}\left(x_{i}, \alpha\right)\right]^{2}}, \\
L_{2, I}[a, b] & =\sqrt{\int_{a}^{b}\left[u_{\text {Exact }}(x, \alpha)-\phi_{i, n}(x, \alpha)\right]^{2} d x}
\end{aligned}
$$

where $n=1,2, \ldots$ represents the number of iterations. Moreover, we give the error residual in the non-linear problem.

The computations for the problems were done with a precision of 40 digits using the Maple 18 package.

\section{Problem 1.}

Let us consider the following linear fuzzy system of Volterra integro-differential equations (LFSVIDEs) of the second kind

$$
\left\{\begin{array}{l}
\widetilde{u_{1}^{\prime \prime}}(x, \alpha)=\widetilde{f_{1}}(x, \alpha)+\int_{0}^{x}\left[\widetilde{u_{1}}(t, \alpha)+(x-t) \widetilde{u_{2}}(t, \alpha)\right] d t, \\
\widetilde{u_{2}^{\prime \prime}}(x, \alpha)=\widetilde{f_{2}}(x, \alpha)+\int_{0}^{x}\left[(x-t) \widetilde{u_{1}}(t, \alpha)-\widetilde{u_{2}}(t, \alpha)\right] d t,
\end{array}\right.
$$

with the initial conditions

$$
\begin{cases}\widetilde{u_{1}}(0, \alpha)=(3-\alpha, 2 \alpha), & \widetilde{u_{1}^{\prime}}(0, \alpha)=\left(2-\alpha, \alpha^{2}\right), \\ \widetilde{u_{2}}(0, \alpha)=(3-2 \alpha, 2 \alpha-1), & \widetilde{u_{2}^{\prime}}(0, \alpha)=\left(4-2 \alpha, 3 \alpha^{2}-\alpha\right),\end{cases}
$$

where $\widetilde{f}_{1}(x, \alpha)=\left[\underline{f_{1}}(x, \alpha), \overline{f_{1}}(x, \alpha)\right]$ and $\widetilde{f}_{2}(x, \alpha)=\left[\underline{f_{2}}(x, \alpha), \overline{f_{2}}(x, \alpha)\right]$ are given by

$$
\begin{aligned}
& \underline{f_{1}}(x, \alpha)=(3-\alpha)+(1-2 \alpha) e^{x}+\left(1-2 \alpha^{3}\right) \cos x, \\
& \overline{f_{1}}(x, \alpha)=\left(5 \alpha^{2}-3\right)-\alpha e^{x}-\left(3 \alpha^{2}-2 \alpha\right) \cos x, \\
& \underline{f_{2}}(x, \alpha)=(3-2 \alpha) x-(1-2 \alpha) e^{x}+\left(2-3 \alpha^{5}\right) \sin x, \\
& \overline{f_{2}}(x, \alpha)=\left(3 \alpha^{5}-2\right) x+\left(2 \alpha^{2}-1\right) e^{x}-\alpha \sin x,
\end{aligned}
$$

The exact solutions of the LFSVIDEs (14) are $\widetilde{u_{1}}(x)=e^{x}+\cos x$ and $\widetilde{u_{2}}(x)=e^{x}+\sin x$.

Operating by the same way proceeding (3)-(9) as above, and applying the fuzzy Standard ADM, the lower iterations (L) are then determined in the following recursive way:

$$
\begin{gathered}
\left\{\begin{array}{l}
\underline{u_{1,0}}(x, \alpha)=(3-\alpha)+(2-\alpha) x+L^{-1} \underline{f_{1}}(x, \alpha), \\
\underline{u_{2,0}}(x, \alpha)=(3-2 \alpha)+(4-2 \alpha) x+L^{-1} \underline{f_{2}}(x, \alpha),
\end{array}\right. \\
\left\{\begin{array}{l}
\underline{u_{1, n+1}}(x, \alpha)=L^{-1}\left[\int_{0}^{x}\left[\underline{u_{1, n}}(t, \alpha)+(x-t) \underline{u_{2, n}}(t, \alpha)\right] d t\right], \quad n=0,1,2, \ldots \\
\underline{u_{2, n+1}}(x, \alpha)=L^{-1}\left[\int_{0}^{x}\left[(x-t) \underline{u_{1, n}}(t, \alpha)-\underline{u_{2, n}}(t, \alpha)\right] d t\right],
\end{array}\right.
\end{gathered}
$$


and the upper iterations (U) are

$$
\begin{gathered}
\left\{\begin{array}{l}
\overline{u_{1,0}}(x, \alpha)=2 \alpha+\alpha^{2} x+L^{-1} \overline{f_{1}}(x, \alpha), \\
\overline{u_{2,0}}(x, \alpha)=(2 \alpha-1)+\left(3 \alpha^{2}-\alpha\right) x+L^{-1} \overline{f_{2}}(x, \alpha),
\end{array}\right. \\
\left\{\begin{array}{l}
\overline{u_{1, n+1}}(x, \alpha)=L^{-1}\left[\int_{0}^{x}\left[\overline{u_{1, n}}(t, \alpha)+(x-t) \overline{u_{2, n}}(t, \alpha)\right] d t\right], \quad n=0,1,2, \ldots \\
\overline{u_{2, n+1}}(x, \alpha)=L^{-1}\left[\int_{0}^{x}\left[(x-t) \overline{u_{1, n}}(t, \alpha)-\overline{u_{2, n}}(t, \alpha)\right] d t\right],
\end{array}\right.
\end{gathered}
$$

Applying the fuzzy Modified Technique (MT) [11], the lower iterations (L) are then determined in the following recursive way:

$$
\begin{gathered}
\left\{\begin{array}{l}
u_{1,0}(x, \alpha)=(3-\alpha)+(2-\alpha) x, \\
\underline{u_{2,0}}(x, \alpha)=(3-2 \alpha)+(4-2 \alpha) x,
\end{array}\right. \\
\left\{\begin{array}{l}
\underline{u_{1,1}}(x, \alpha)=L^{-1} \underline{f_{1}}(x, \alpha)+L^{-1}\left[\int_{0}^{x}\left[\underline{u_{1,0}}(t, \alpha)+(x-t) \underline{u_{2,0}}(t, \alpha)\right] d t\right], \\
\underline{u_{2,1}}(x, \alpha)=L^{-1} \underline{f_{2}}(x, \alpha)+L^{-1}\left[\int_{0}^{x}\left[(x-t) \underline{u_{1,0}}(t, \alpha)-\underline{u_{2,0}}(t, \alpha)\right] d t\right],
\end{array}\right. \\
\left\{\begin{array}{l}
\left.\left.\underline{u_{1, n+1}}(x, \alpha)=L^{-1}\left[\int_{0}^{x}\left[\underline{u_{1, n}}(t, \alpha)+(x-t) \underline{u_{2, n}}(t, \alpha)\right] d t\right], \quad \underline{\underline{u_{2, n}}}(t, \alpha)\right] d t\right], \\
\underline{u_{2, n+1}}(x, \alpha)=L^{-1}\left[\int _ { 0 } ^ { x } \left[(x-t) \underline{u_{1, n}}(t, \alpha), \ldots, 3, \ldots\right.\right.
\end{array}\right.
\end{gathered}
$$

and the upper iterations (U) are

$$
\begin{gathered}
\left\{\begin{array}{l}
\overline{u_{1,0}}(x, \alpha)=2 \alpha+\alpha^{2} x, \\
\overline{u_{2,0}}(x, \alpha)=(2 \alpha-1)+\left(3 \alpha^{2}-\alpha\right) x,
\end{array}\right. \\
\left\{\begin{array}{l}
\overline{u_{1,1}}(x, \alpha)=L^{-1} \overline{f_{1}}(x, \alpha)+L^{-1}\left[\int_{0}^{x}\left[\overline{u_{1,0}}(t, \alpha)+(x-t) \overline{u_{2,0}}(t, \alpha)\right] d t\right], \\
\overline{u_{2,1}}(x, \alpha)=L^{-1} \overline{f_{2}}(x, \alpha)+L^{-1}\left[\int_{0}^{x}\left[(x-t) \overline{u_{1,0}}(t, \alpha)-\overline{u_{2,0}}(t, \alpha)\right] d t\right],
\end{array}\right. \\
\left\{\begin{array}{l}
\overline{u_{1, n+1}}(x, \alpha)=L^{-1}\left[\int_{0}^{x}\left[\overline{u_{1, n}}(t, \alpha)+(x-t) \overline{u_{2, n}}(t, \alpha)\right] d t\right], \quad n=1,2,3, \ldots \\
\overline{u_{2, n+1}}(x, \alpha)=L^{-1}\left[\int_{0}^{x}\left[(x-t) \overline{u_{1, n}}(t, \alpha)-\overline{u_{2, n}}(t, \alpha)\right] d t\right],
\end{array}\right.
\end{gathered}
$$

In Tables 1-4 we shown the maximum errors between the exact solutions and by fuzzy standard ADM and fuzzy MT for different norms on the interval $[0,1]$, where $n$ represents the number of iterations.

Table 1. Norm Error for $\widetilde{u_{1}}(x, \alpha)$ (Problem 1) when $\alpha=0.3$

\begin{tabular}{cccccccc}
\hline & \multicolumn{4}{c}{$L_{\infty}[0,1]$} & \multicolumn{2}{c}{$L_{2, S}[0,1]$} & \multicolumn{2}{c}{$L_{2, I}[0,1]$} \\
\hline$n$ & $i$ & $\mathrm{ADM}$ & $\mathrm{MT}$ & $\mathrm{ADM}$ & $\mathrm{MT}$ & $\mathrm{ADM}$ & $\mathrm{MT}$ \\
\hline 3 & $L$ & $8.861 E-06$ & $1.112 E-04$ & $9.560 E-06$ & $1.224 E-04$ & $2.017 E-06$ & $2.681 E-05$ \\
& $U$ & $1.499 E-06$ & $6.271 E-05$ & $1.620 E-06$ & $6.916 E-05$ & $3.435 E-07$ & $1.519 E-05$ \\
4 & $L$ & $2.637 E-08$ & $1.289 E-07$ & $4.215 E-08$ & $1.428 E-07$ & $1.177 E-08$ & $3.098 E-08$ \\
& $U$ & $1.547 E-08$ & $8.198 E-08$ & $3.198 E-08$ & $9.351 E-08$ & $9.480 E-09$ & $2.103 E-08$ \\
5 & $L$ & $2.035 E-08$ & $2.040 E-08$ & $3.765 E-08$ & $3.768 E-08$ & $1.095 E-08$ & $1.096 E-08$ \\
& $U$ & $1.672 E-08$ & $1.675 E-08$ & $3.280 E-8$ & $3.282 E-08$ & $9.631 E-09$ & $9.634 E-09$ \\
\hline
\end{tabular}


Table 2. Norm Error for $\widetilde{u_{2}}(x, \alpha)$ (Problem 1) when $\alpha=0.3$

\begin{tabular}{cccccccc}
\hline & \multicolumn{4}{c}{$L_{\infty}[0,1]$} & \multicolumn{2}{c}{$L_{2, S}[0,1]$} & \multicolumn{2}{c}{$L_{2, I}[0,1]$} \\
\hline$n$ & $i$ & $\mathrm{ADM}$ & $\mathrm{MT}$ & $\mathrm{ADM}$ & $\mathrm{MT}$ & $\mathrm{ADM}$ & $\mathrm{MT}$ \\
\hline 3 & $L$ & $6.781 E-06$ & $5.881 E-06$ & $7.323 E-06$ & $6.567 E-06$ & $1.549 E-06$ & $1.472 E-06$ \\
& $U$ & $1.305 E-06$ & $2.815 E-05$ & $1.407 E-06$ & $3.087 E-05$ & $2.961 E-07$ & $6.720 E-06$ \\
4 & $L$ & $3.318 E-08$ & $4.353 E-08$ & $5.997 E-08$ & $6.799 E-08$ & $1.729 E-08$ & $1.877 E-08$ \\
& $U$ & $5.533 E-09$ & $1.646 E-08$ & $1.044 E-08$ & $1.087 E-08$ & $2.975 E-09$ & $3.614 E-09$ \\
5 & $L$ & $2.757 E-08$ & $2.757 E-08$ & $5.616 E-08$ & $5.616 E-08$ & $1.660 E-08$ & $1.660 E-08$ \\
& $U$ & $4.671 E-08$ & $4.683 E-09$ & $9.892 E 09$ & $9.898 E-09$ & $2.876 E-09$ & $2.877 E-09$ \\
\hline
\end{tabular}

Table 3. Norm Error for $\widetilde{u_{1}}(x, \alpha)$ (Problem 1) when $\alpha=0.9$

\begin{tabular}{cccccccc}
\hline & \multicolumn{2}{c}{$L_{\infty}[0,1]$} & \multicolumn{2}{c}{$L_{2, S}[0,1]$} & \multicolumn{2}{c}{$L_{2, I}[0,1]$} \\
\cline { 1 - 7 }$n$ & $i$ & $\mathrm{ADM}$ & $\mathrm{MT}$ & $\mathrm{ADM}$ & $\mathrm{MT}$ & $\mathrm{ADM}$ & $\mathrm{MT}$ \\
\hline 3 & $L$ & $6.565 E-06$ & $2.533 E-05$ & $7.086 E-06$ & $2.783 E-05$ & $1.496 E-06$ & $6.075 E-06$ \\
& $U$ & $5.477 E-06$ & $9.163 E-06$ & $5.912 E-06$ & $1.018 E-05$ & $1.248 E-06$ & $20264 E-06$ \\
4 & $L$ & $1.009 E-08$ & $3.163 E-08$ & $1.427 E-08$ & $3.544 E-08$ & $3.781 E-09$ & $7.792 E-09$ \\
& $U$ & $3.448 E-09$ & $1.362 E-08$ & $7.534 E-09$ & $1.711 E-08$ & $2.384 E-09$ & $4.220 E-09$ \\
5 & $L$ & $5.478 E-09$ & $5.488 E-09$ & $1.068 E-08$ & $1.068 E-08$ & $3.139 E-09$ & $3.140 E-09$ \\
& $U$ & $4.725 E-09$ & $4.731 E-09$ & $9.424 E-09$ & $9.428 E-09$ & $2.774 E-09$ & $7.792 E-09$ \\
\hline
\end{tabular}

Table 4. Norm Error for $\widetilde{u_{2}}(x, \alpha)$ (Problem 1) when $\alpha=0.9$

\begin{tabular}{cccccccc}
\hline & \multicolumn{2}{c}{$L_{\infty}[0,1]$} & \multicolumn{2}{c}{$L_{2, S}[0,1]$} & \multicolumn{2}{c}{$L_{2, I}[0,1]$} \\
\hline$n$ & $i$ & $\mathrm{ADM}$ & $\mathrm{MT}$ & $\mathrm{ADM}$ & $\mathrm{MT}$ & $\mathrm{ADM}$ & $\mathrm{MT}$ \\
\hline 3 & $L$ & $3.348 E-06$ & $2.314 E-05$ & $3.617 E-06$ & $2.545 E-05$ & $7.657 E-07$ & $5.565 E-06$ \\
& $U$ & $2.144 E-06$ & $1.220 E-05$ & $2.320 E-06$ & $1.352 E-05$ & $4.492 E-07$ & $2.996 E-06$ \\
4 & $L$ & $1.216 E-07$ & $1.118 E-8$ & $2.087 E-08$ & $1.733 E-8$ & $5.934 E-09$ & $4.481 E-09$ \\
& $U$ & $8.131 E-10$ & $1.502 E-8$ & $1.572 E-09$ & $1.609 E-08$ & $4.182 E-10$ & $3.314 E-09$ \\
5 & $L$ & $9.265 E-09$ & $9.276 E-09$ & $1.887 E-08$ & $1.887 E-08$ & $5.575 E-09$ & $5.576 E-09$ \\
& $U$ & $1.130 E-09$ & $1.123 E-09$ & $1.982 E-09$ & $1.978 E-09$ & $5.553 E-10$ & $5.545 E-10$ \\
\hline
\end{tabular}

In the following Figures (1-12) shows both the exact solutions of $\left(\widetilde{u_{i}}(x, \alpha), i=1,2\right)$ and the Standard $\operatorname{ADM}\left(\phi_{i, 3}(x), i=1,2\right)$ for the interval $0 \leq t \leq 1$. We represent the exact solutions with a continuous line and use the symbol $\circ$ for the $\operatorname{ADM} \phi_{i, 3}(x), i=1,2$. 


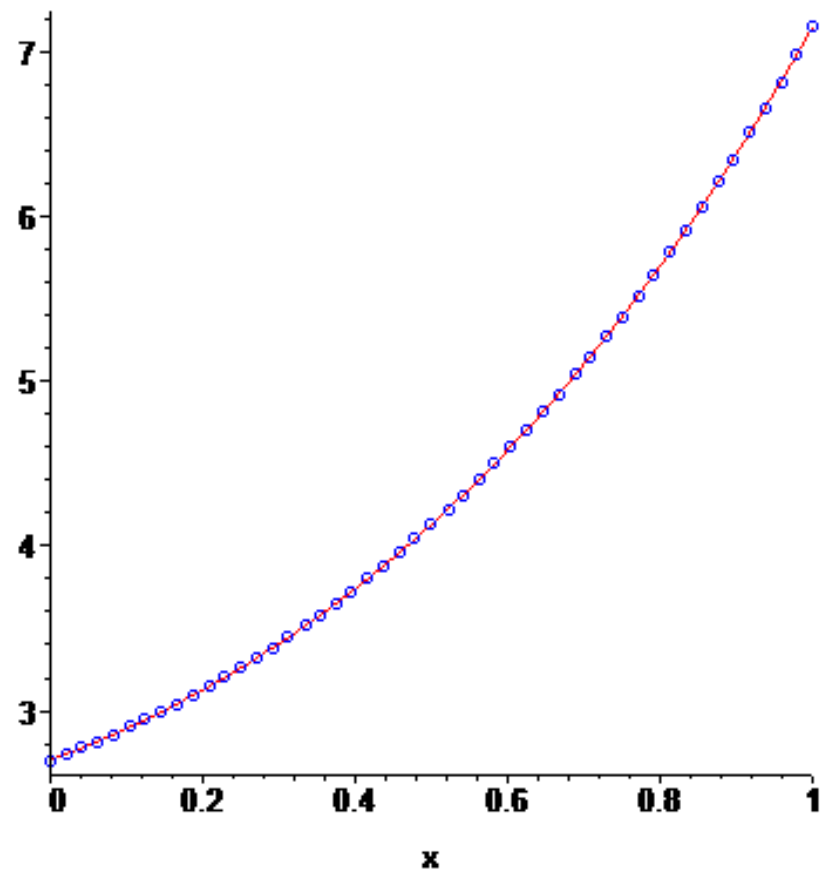

Figure 1: $\underline{\phi_{1,3}}(x, \alpha), \underline{u_{1}}(x ., \alpha), \alpha=0.3$

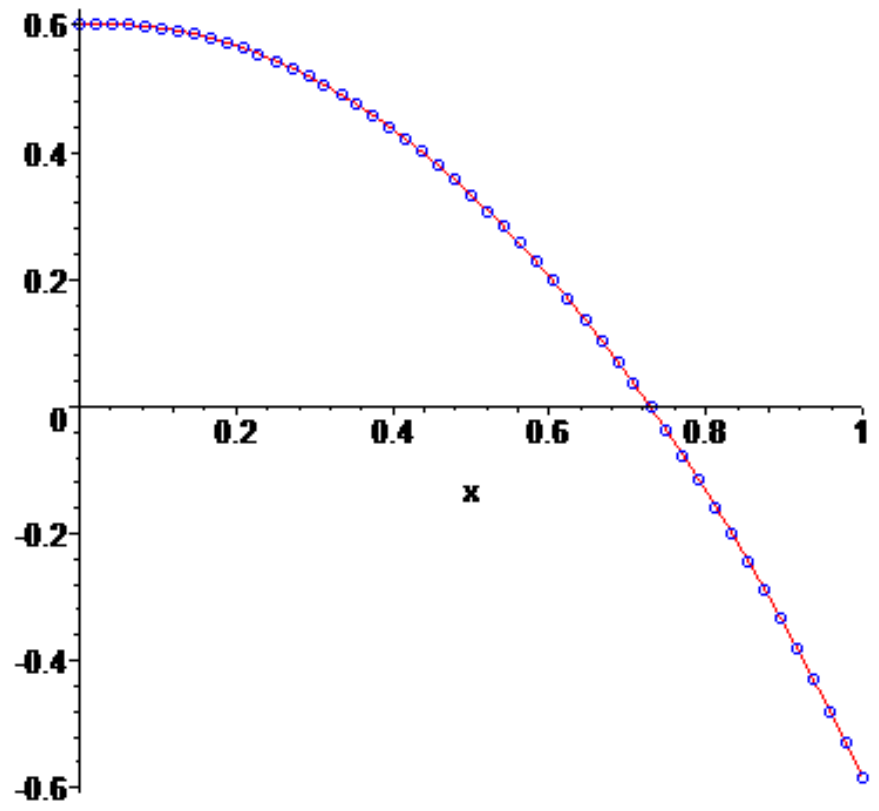

Figure 2: $\overline{\phi_{1,3}}(x, \alpha), \overline{u_{1}}(x, \alpha), \alpha=0.3$ 


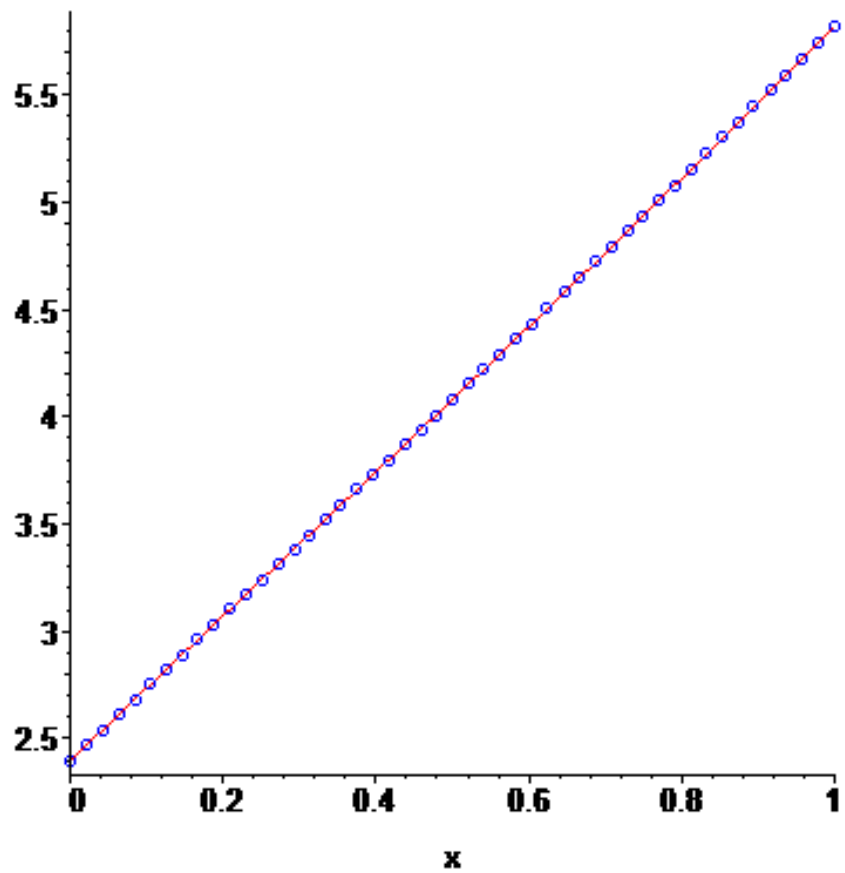

Figure 3: $\phi_{2,3}(x, \alpha), \underline{u_{2}}(x, \alpha), \alpha=0.3$

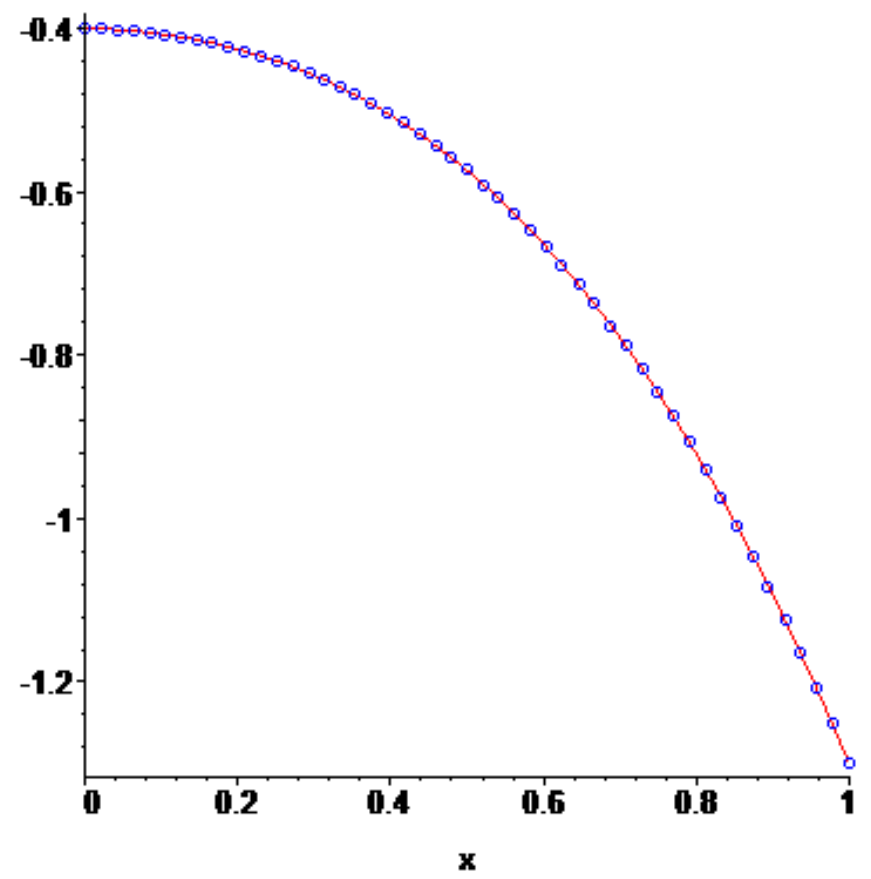

Figure 4: $\overline{\phi_{2,3}}(x, \alpha), \overline{u_{2}}(x, \alpha), \alpha=0.3$ 


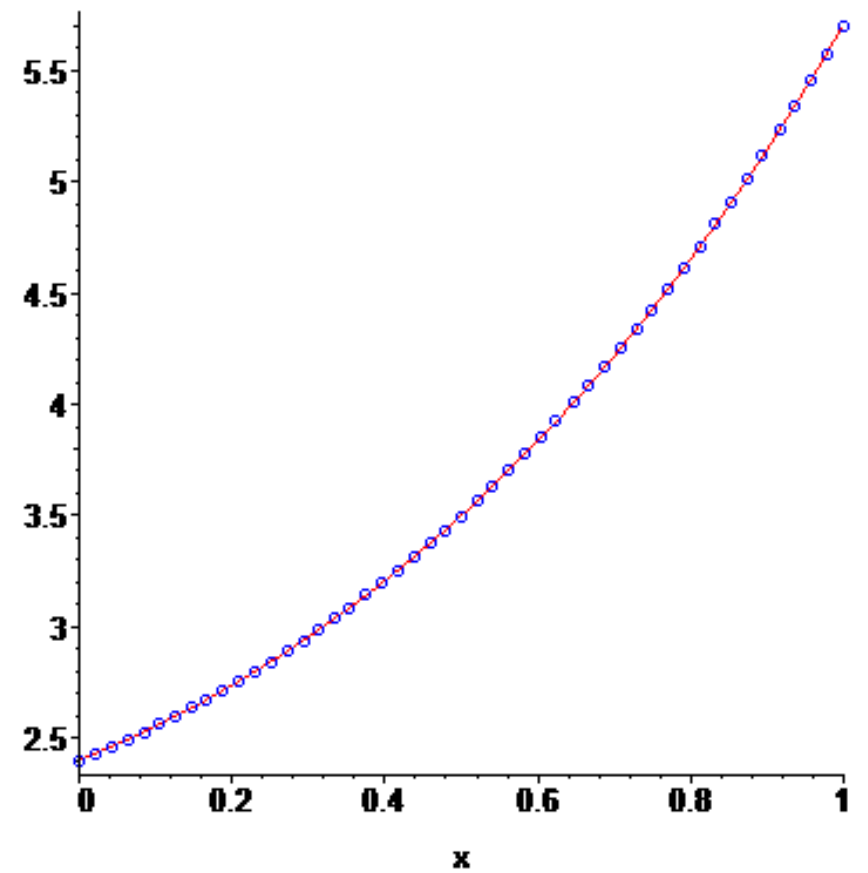

Figure 5: $\phi_{1,3}(x, \alpha), \underline{u_{1}}(x, \alpha), \alpha=0.6$

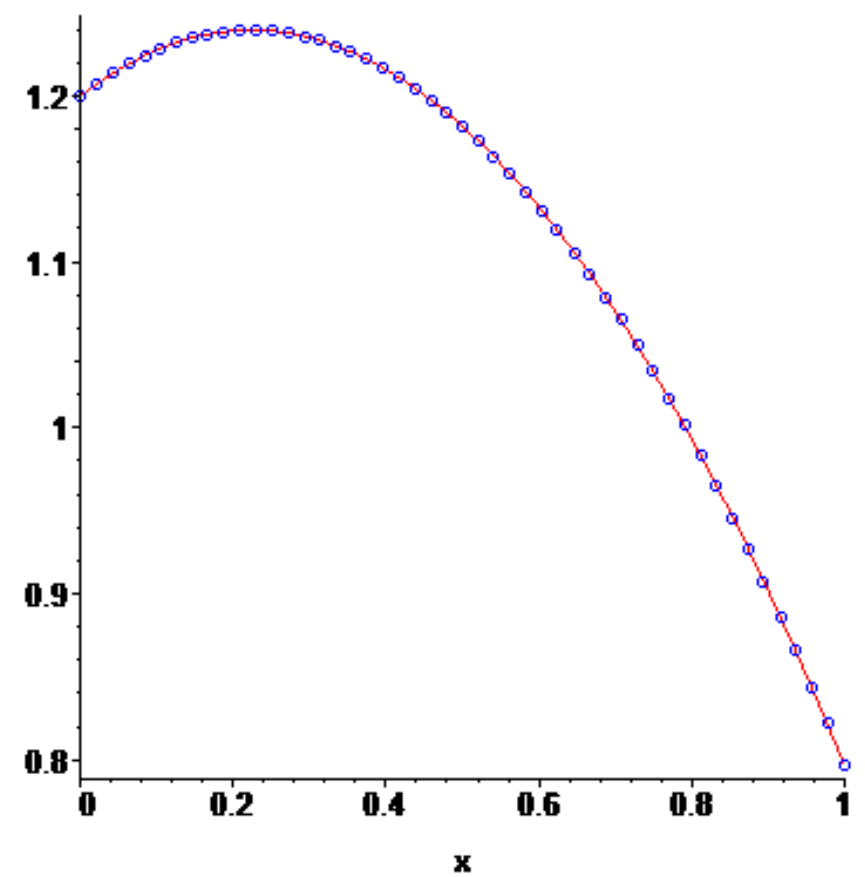

Figure 6: $\overline{\phi_{1,3}}(x, \alpha), \overline{u_{1}}(x, \alpha), \alpha=0.6$ 


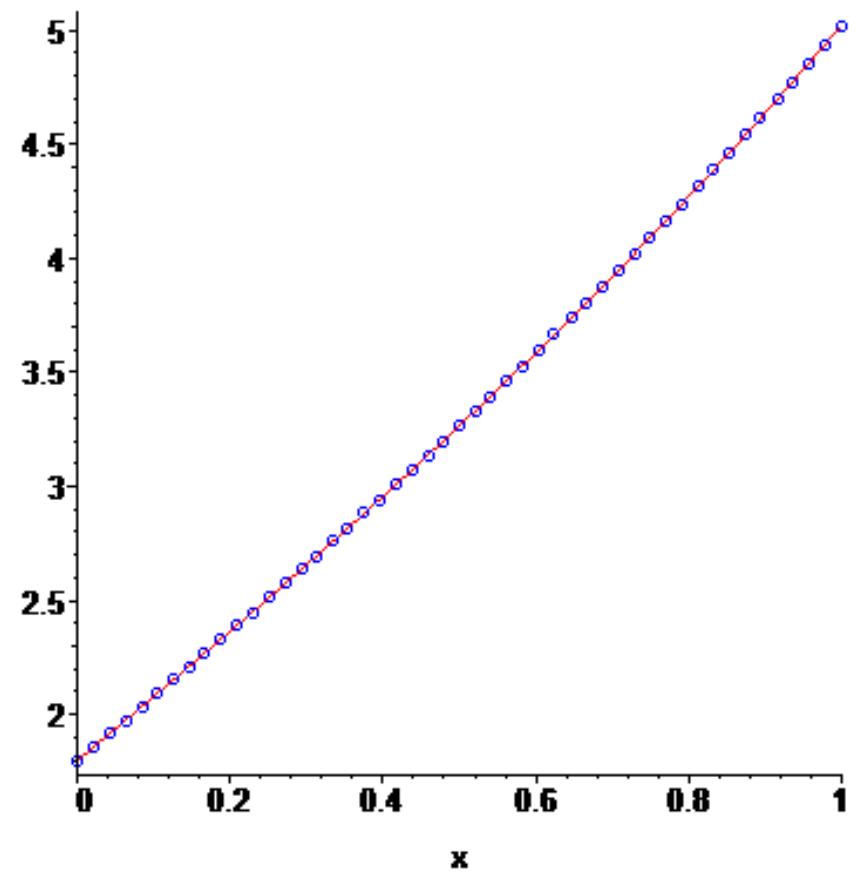

Figure 7: $\phi_{2,3}(x, \alpha), \underline{u_{2}}(x, \alpha), \alpha=0.6$

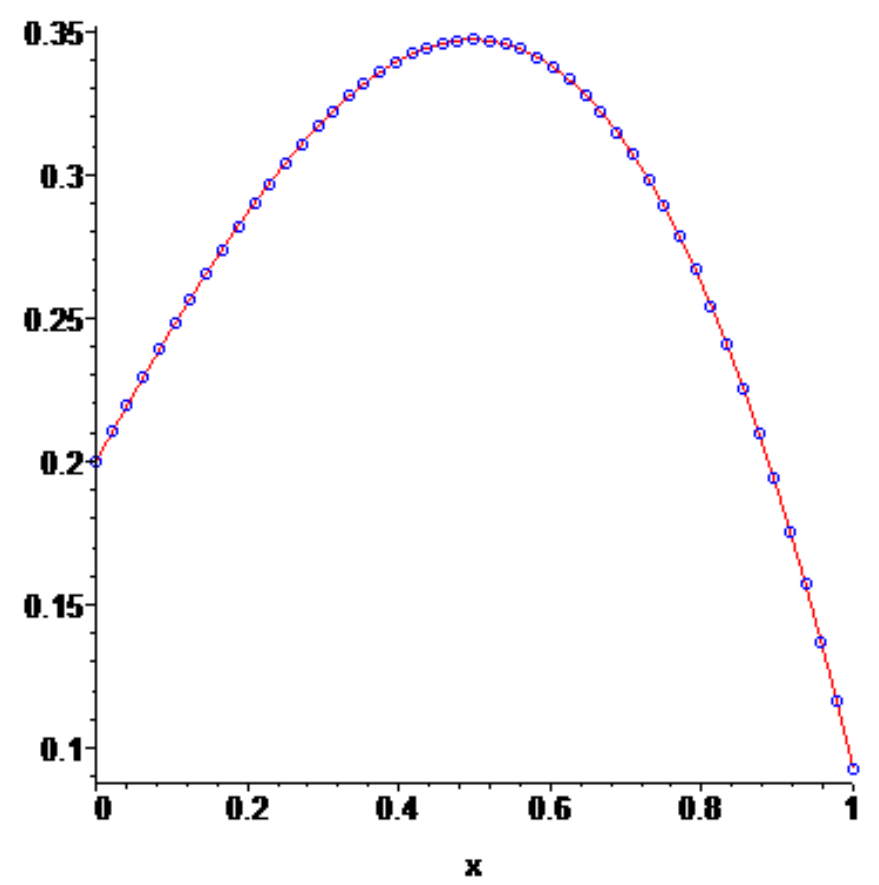

Figure 8: $\overline{\phi_{2,3}}(x, \alpha), \overline{u_{2}}(x, \alpha), \alpha=0.6$ 


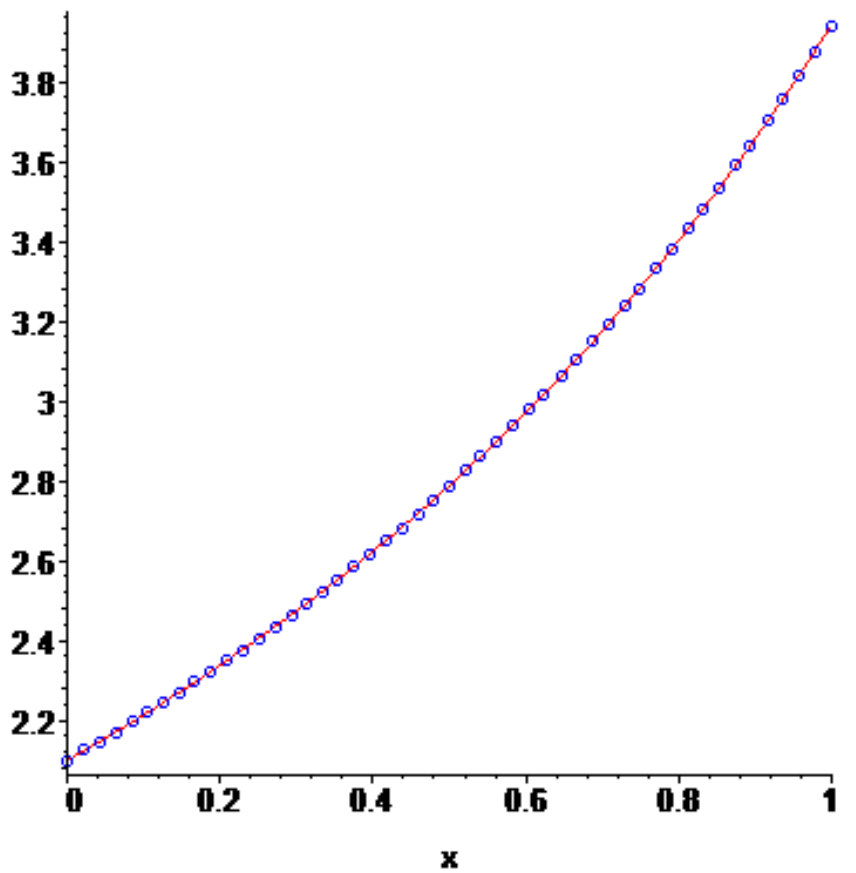

Figure 9: $\phi_{1,3}(x, \alpha), \underline{u_{1}}(x, \alpha), \alpha=0.9$

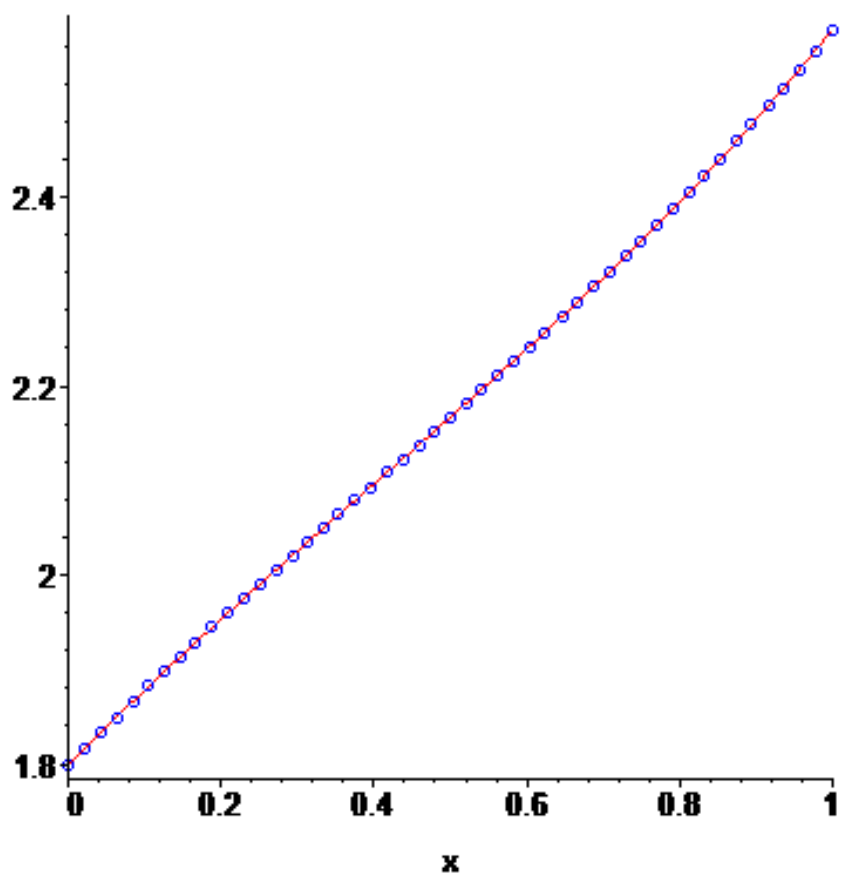

Figure 10: $\overline{\phi_{1,3}}(x, \alpha), \overline{u_{1}}(x, \alpha), \alpha=0.9$ 


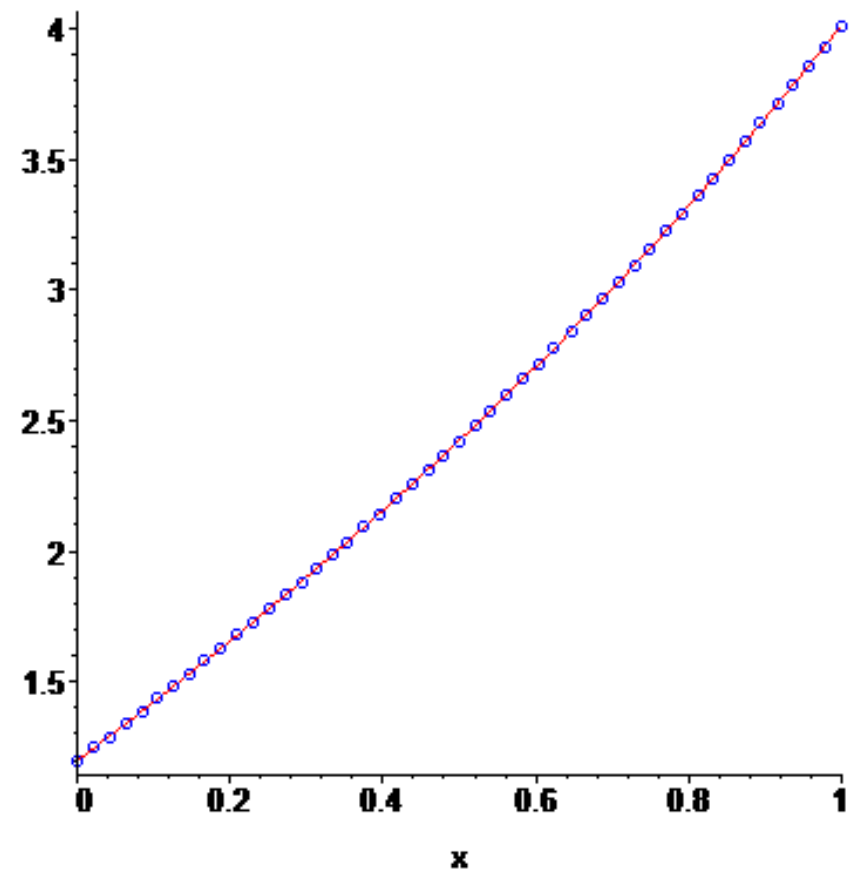

Figure 11: $\underline{\phi_{2,3}}(x, \alpha), \underline{u_{2}}(x, \alpha), \alpha=0.9$

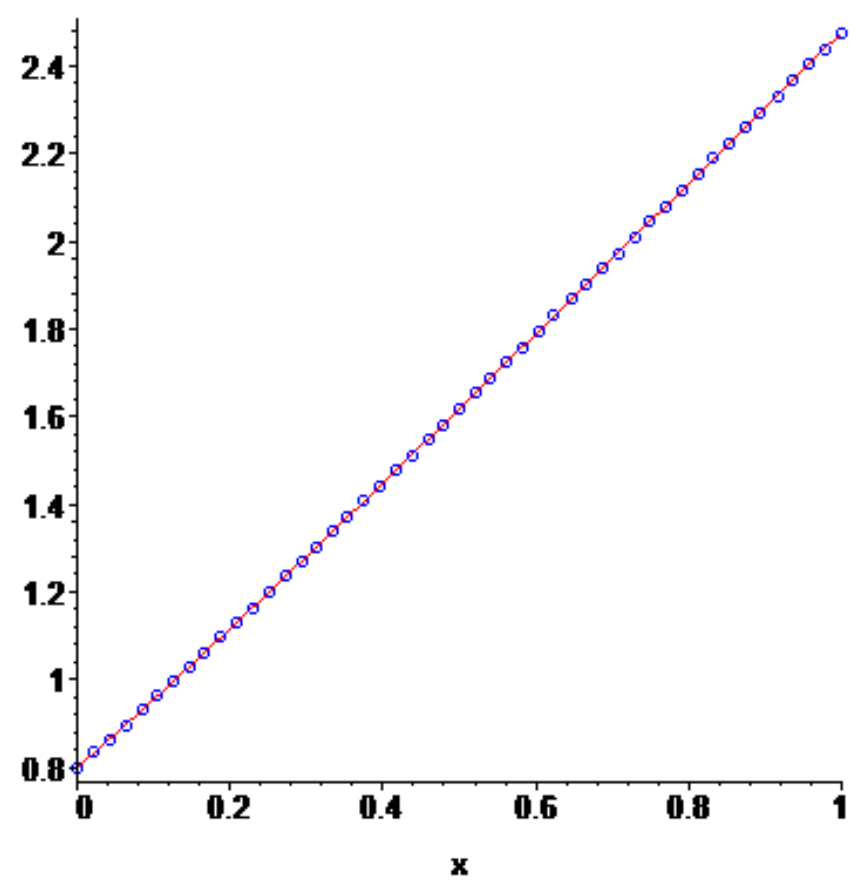

Figure 12: $\overline{\phi_{2,3}}(x, \alpha), \overline{u_{2}}(x, \alpha), \alpha=0.9$ 


\section{Problem 2.}

We consider the following non-linear fuzzy system of Volterra integro-differential equations (NFSVIDEs) of the second kind

$$
\left\{\begin{array}{l}
\widetilde{u_{1}^{\prime \prime}}(x, \alpha)=\widetilde{f_{1}}(x, \alpha)+\int_{0}^{x}\left[\widetilde{u_{1}^{2}}(t, \alpha)+\widetilde{u_{2}^{2}}(t, \alpha)\right] d t \\
\widetilde{u_{2}^{\prime \prime}}(x, \alpha)=\widetilde{f_{2}}(x, \alpha)+\int_{0}^{x}\left[\widetilde{u_{1}^{2}}(t, \alpha)-\widetilde{u_{2}^{2}}(t, \alpha)\right] d t
\end{array}\right.
$$

with the initial conditions

$$
\left\{\begin{array}{l}
\widetilde{u_{1}}(0, \alpha)=(0,0), \widetilde{u_{1}^{\prime}}(0, \alpha)=(2-\alpha, \alpha), \\
\widetilde{u_{2}}(0, \alpha)=(0,0), \widetilde{u_{2}^{\prime}}(0, \alpha)=\left(2 \alpha-\alpha^{2}, 3-2 \alpha\right),
\end{array}\right.
$$

where $\widetilde{f}_{1}(x, \alpha)=\left[\underline{f_{1}}(x, \alpha), \overline{f_{1}}(x, \alpha)\right]$ and $\widetilde{f}_{2}(x, \alpha)=\left[\underline{f_{2}}(x, \alpha), \overline{f_{2}}(x, \alpha)\right]$ are given by

$$
\begin{aligned}
& \underline{f_{1}}(x, \alpha)=(9-3 \alpha) x+\frac{\left(1-3 \alpha^{2}\right)}{3} x^{3}+\frac{(2-4 \alpha)}{7} x^{7}, \\
& \overline{f_{1}}(x, \alpha)=\left(1+5 \alpha^{2}\right) x-\frac{\left(\alpha^{3}+3\right)}{6} x^{3}-\frac{\left(\alpha^{2}+1\right)}{7} x^{7}, \\
& \underline{f_{2}}(x, \alpha)=-\left(7-\alpha^{2}\right) x-\frac{\left(17 \alpha^{2}-\alpha\right)}{20} x^{5}, \\
& \overline{f_{2}}(x, \alpha)=-(2+4 \alpha) x-\frac{4 \alpha}{5} x^{5},
\end{aligned}
$$

The exact solutions of the NFSVIDEs $(19)$ are $\widetilde{u_{1}}(x)=x+x^{3}$ and $\widetilde{u_{2}}(x)=x-x^{3}$.

Operating by the same way proceeding (3)-(9) as above, and applying the fuzzy Standard $\mathrm{ADM}$, the lower iterations $(\mathrm{L})$ are then determined in the following recursive way:

$$
\begin{gathered}
\left\{\begin{array}{l}
\underline{u_{1,0}}(x, \alpha)=(2-\alpha) x+L^{-1} \underline{f_{1}}(x, \alpha), \\
\underline{u_{2,0}}(x, \alpha)=\left(2 \alpha-\alpha^{2}\right) x+L^{-1} \underline{f_{2}}(x, \alpha),
\end{array}\right. \\
\left\{\begin{array}{l}
\underline{u_{1, n+1}}(x, \alpha)=L^{-1}\left[\int_{0}^{x}\left(\underline{A_{1, n}}+\underline{A_{2, n}}\right) d t\right], \quad n=0,1,2, \ldots \\
\underline{u_{2, n+1}}(x, \alpha)=L^{-1}\left[\int_{0}^{x}\left(\underline{A_{1, n}}-\underline{A_{2, n}}\right) d t\right],
\end{array}\right.
\end{gathered}
$$

and the upper iterations (U) are

$$
\begin{gathered}
\left\{\begin{array}{l}
\overline{u_{1,0}}(x, \alpha)=\alpha x+L^{-1} \overline{f_{1}}(x, \alpha), \\
\overline{u_{2,0}}(x, \alpha)=(3-2 \alpha) x+L^{-1} \overline{f_{2}}(x, \alpha),
\end{array}\right. \\
\left\{\begin{array}{l}
\overline{u_{1, n+1}}(x, \alpha)=L^{-1}\left[\int_{0}^{x}\left(\overline{A_{1, n}}+\overline{A_{2, n}}\right) d t\right], \quad n=0,1,2, \ldots \\
\overline{u_{2, n+1}}(x, \alpha)=L^{-1}\left[\int_{0}^{x}\left(\overline{A_{1, n}}-\overline{A_{2, n}}\right) d t\right],
\end{array}\right.
\end{gathered}
$$


where the non-linear terms defined by the series

$$
\begin{aligned}
& \underline{u_{1, n}^{2}}(t, \alpha)=\sum_{n=0}^{\infty} \underline{A_{1, n}}, \quad \overline{u_{1, n}^{2}}(t, \alpha)=\sum_{n=0}^{\infty} \overline{A_{1, n}}, \\
& \underline{u_{2, n}^{2}}(t, \alpha)=\sum_{n=0}^{\infty} \underline{A_{2, n}}, \quad \overline{u_{2, n}^{2}}(t, \alpha)=\sum_{n=0}^{\infty} \overline{A_{2, n}},
\end{aligned}
$$

the corresponding Adomian polynomials $\left[\underline{A_{1, n}}, \overline{A_{1, n}}\right]$ and $\left[\underline{A_{2, n}}, \overline{A_{2, n}}\right][3,4]$ are

$$
\begin{aligned}
& \underline{A_{1, n}}=\sum_{n=0}^{\infty} \frac{u_{1, i} u_{1, n-i}}{}, \quad \overline{A_{1, n}}=\sum_{n=0}^{\infty} \overline{u_{1, i} u_{1, n-i}}, \quad n \geq i, \quad n=0,1,2, \ldots \\
& \underline{A_{2, n}}=\sum_{n=0}^{\infty} \frac{u_{2, i} u_{2, n-i}}{}, \quad \overline{A_{2, n}}=\sum_{n=0}^{\infty} \overline{u_{2, i} u_{2, n-i}}, \quad n \geq i, \quad n=0,1,2, \ldots
\end{aligned}
$$

Applying the fuzzy Modified Technique (MT) [11], the lower iterations (L) are then determined in the following recursive way:

$$
\begin{gathered}
\left\{\begin{array}{l}
\underline{u_{1,0}}(x, \alpha)=(2-\alpha) x, \\
\underline{u_{2,0}}(x, \alpha)=\left(2 \alpha-\alpha^{2}\right) x,
\end{array}\right. \\
\left\{\begin{array}{l}
\underline{u_{1,1}}(x, \alpha)=L^{-1} \underline{f_{1}}(x, \alpha)+L^{-1}\left[\int_{0}^{x}\left(\underline{A_{1,0}}+\underline{A_{2,0}}\right) d t\right], \\
\underline{u_{2,1}}(x, \alpha)=L^{-1} \underline{f_{2}}(x, \alpha)+L^{-1}\left[\int_{0}^{x}\left(\underline{A_{1,0}}-\underline{A_{2,0}}\right) d t,\right.
\end{array}\right. \\
\left\{\begin{array}{l}
\underline{u_{1, n+1}}(x, \alpha)=L^{-1}\left[\int_{0}^{x}\left(\underline{A_{1, n}}+\underline{A_{2, n}}\right) d t\right], \quad n=1,2,3, \ldots \\
\underline{u_{2, n+1}}(x, \alpha)=L^{-1}\left[\int_{0}^{x}\left(\underline{A_{1, n}}-\underline{A_{2, n}}\right) d t\right],
\end{array}\right.
\end{gathered}
$$

and the upper iterations (U) are

$$
\begin{gathered}
\left\{\begin{array}{l}
\overline{u_{1,0}}(x, \alpha)=\alpha x, \\
\overline{u_{2,0}}(x, \alpha)=(3-2 \alpha) x,
\end{array}\right. \\
\left\{\begin{array}{l}
\overline{u_{1,1}}(x, \alpha)=L^{-1} \overline{f_{1}}(x, \alpha)+L^{-1}\left[\int_{0}^{x}\left(\overline{A_{1,0}}+\overline{A_{2,0}}\right) d t\right], \\
\overline{u_{2,1}}(x, \alpha)=L^{-1} \overline{f_{2}}(x, \alpha)+L^{-1}\left[\int_{0}^{x}\left(\overline{A_{1,0}}-\overline{A_{2,0}}\right) d t\right],
\end{array}\right. \\
\left\{\begin{array}{l}
\overline{u_{1, n+1}}(x, \alpha)=L^{-1}\left[\int_{0}^{x}\left(\overline{A_{1, n}}+\overline{A_{2, n}}\right) d t\right], \quad n=1,2,3, \ldots \\
\overline{u_{2, n+1}}(x, \alpha)=L^{-1}\left[\int_{0}^{x}\left(\overline{A_{1, n}}-\overline{A_{2, n}}\right) d t\right],
\end{array}\right.
\end{gathered}
$$

In Tables 5-8 display a comparison of the numerical results applying the ADM $(n=4)$, Iteration of the Integral Equation (IIE) (23), (24) and the numerical solution of (23), (24) with the Simpson rule (SIMP) and the trapezoidal rule (TRAP) on the interval $[0,1]$. Twenty points have been used in the Simpson and trapezoidal methods. In Tables 9 and 10 we present the maximum error residual obtained by fuzzy standard ADM and fuzzy MT on the interval $[0,1]$, where $n$ represents the number of iterations. 
Table 5. Numerical results for $\widetilde{u_{1}}(x, \alpha)$ (Problem 2) when $\alpha=0.3$

\begin{tabular}{cccccc}
\hline$x$ & $i$ & ADM & IIE & SIMP & TRAP \\
\hline 0.0 & $L$ & 0.0 & 0.0 & 0.0 & 0.0 \\
& $U$ & 0.0 & 0.0 & 0.0 & 0.0 \\
0.2 & $L$ & 0.350820906 & 0.350820906 & 0.350820906 & 0.350820978 \\
& $U$ & 0.061956312 & 0.061956312 & 0.061956312 & 0.061956441 \\
0.4 & $L$ & 0.767091047 & 0.767091047 & 0.767091047 & 0.767093546 \\
& $U$ & 0.136187326 & 0.136187326 & 0.136187326 & 0.136191319 \\
0.6 & $L$ & 1.317168717 & 1.317168717 & 1.317168718 & 1.317190739 \\
& $U$ & 0.237475634 & 0.237475634 & 0.237475634 & 0.237504395 \\
0.8 & $L$ & 2.076937697 & 2.076937698 & 2.076937706 & 2.077053136 \\
& $U$ & 0.384710630 & 0.384710628 & 0.384710630 & 0.384822684 \\
1.0 & $L$ & 3.139469824 & 3.139469864 & 3.139469904 & 3.139936553 \\
& $U$ & 0.600309591 & 0.600309524 & 0.600309527 & 0.600616856 \\
\hline
\end{tabular}

Table 6. Numerical results for $\widetilde{u_{2}}(x, \alpha)$ (Problem 2) when $\alpha=0.3$

\begin{tabular}{cccccc}
\hline$x$ & $i$ & ADM & IIE & SIMP & TRAP \\
\hline 0.0 & $L$ & 0.0 & 0.0 & 0.0 & 0.0 \\
& $U$ & 0.0 & 0.0 & 0.0 & 0.0 \\
0.2 & $L$ & 0.092801026 & 0.092801026 & 0.092801026 & 0.092801087 \\
& $U$ & 0.475703185 & 0.475703185 & 0.475703185 & 0.475703060 \\
0.4 & $L$ & 0.130785108 & 0.130785108 & 0.130785108 & 0.130787377 \\
& $U$ & 0.924910864 & 0.924910864 & 0.924910864 & 0.924907020 \\
0.6 & $L$ & 0.061390455 & 0.061390455 & 0.061390456 & 0.061411601 \\
& $U$ & 1.317657321 & 1.317657321 & 1.317657320 & 1.317629935 \\
0.8 & $L$ & -0.161630983 & -0.161630982 & -0.161630975 & -0.161517388 \\
& $U$ & 1.617533199 & 1.617533199 & 1.617533198 & 1.617428686 \\
1.0 & $L$ & -0.570121458 & -0.570121448 & -0.570121413 & -0.569668950 \\
& $U$ & 1.779759247 & 1.779759262 & 1.779759263 & 1.779483691 \\
\hline
\end{tabular}


Table 7. Numerical results for $\widetilde{u_{1}}(x, \alpha)$ (Problem 2) when $\alpha=0.9$

\begin{tabular}{cccccc}
\hline$x$ & $i$ & ADM & IIE & SIMP & TRAP \\
\hline 0.0 & $L$ & 0.0 & 0.0 & 0.0 & 0.0 \\
& $U$ & 0.0 & 0.0 & 0.0 & 0.0 \\
0.2 & $L$ & 0.228404070 & 0.228404070 & 0.228404070 & 0.228404119 \\
& $U$ & 0.186735344 & 0.186735344 & 0.186735344 & 0.186735394 \\
0.4 & $L$ & 0.507332123 & 0.507332123 & 0.507332123 & 0.507333716 \\
& $U$ & 0.413926676 & 0.413926676 & 0.413926676 & 0.413928238 \\
0.6 & $L$ & 0.887832878 & 0.887832878 & 0.887832879 & 0.887845675 \\
& $U$ & 0.722198170 & 0.722198170 & 0.722198171 & 0.722209943 \\
0.8 & $L$ & 1.422181142 & 1.422181142 & 1.422181145 & 1.422242142 \\
& $U$ & 1.152251625 & 1.152251626 & 1.152251628 & 1.152302769 \\
1.0 & $L$ & 2.165166483 & 2.165166491 & 2.165166509 & 2.165395782 \\
& $U$ & 1.744176060 & 1.744176064 & 1.744176075 & 1.744349048 \\
\hline
\end{tabular}

Table 8. Numerical results for $\widetilde{u_{2}}(x, \alpha)$ (Problem 2) when $\alpha=0.9$

\begin{tabular}{cccccc}
\hline$x$ & $i$ & ADM & IIE & SIMP & TRAP \\
\hline 0.0 & $L$ & 0.0 & 0.0 & 0.0 & 0.0 \\
& $U$ & 0.0 & 0.0 & 0.0 & 0.0 \\
0.2 & $L$ & 0.189747962 & 0.189747962 & 0.189747962 & 0.189747969 \\
& $U$ & 0.232529982 & 0.232529982 & 0.232529982 & 0.232529970 \\
0.4 & $L$ & 0.330021457 & 0.330021457 & 0.330021457 & 0.330021918 \\
& $U$ & 0.420160289 & 0.420160289 & 0.420160289 & 0.420160096 \\
0.6 & $L$ & 0.371610554 & 0.371610554 & 0.371610555 & 0.371616888 \\
& $U$ & 0.517601598 & 0.517601598 & 0.517601598 & 0.517602534 \\
0.8 & $L$ & 0.266194321 & 0.266194321 & 0.266194325 & 0.266237859 \\
& $U$ & 0.478812319 & 0.478812319 & 0.478812322 & 0.478830230 \\
1.0 & $L$ & -0.032275868 & -0.032275864 & -0.032275846 & -0.032075986 \\
& $U$ & 0.256634701 & 0.256634705 & 0.256634717 & 0.256743055 \\
\hline
\end{tabular}

Table 9. Error Residual for $\widetilde{u_{1}}(x, \alpha)$ (Problem 2)

\begin{tabular}{cccccccc}
\hline & \multicolumn{4}{c}{$\alpha=0.3$} & \multicolumn{2}{c}{$\alpha=0.6$} & \multicolumn{2}{c}{$\alpha=0.9$} \\
\hline$n$ & $i$ & ADM & MT & ADM & MT & ADM & MT \\
\hline 3 & $L$ & $1.128 E-03$ & $4.673 E-01$ & $6.123 E-04$ & $3.920 E-01$ & $2.906 E-04$ & $3.152 E-01$ \\
& $U$ & $1.600 E-03$ & $7.019 E-02$ & $4.431 E-04$ & $1.227 E-01$ & $1.895 E-04$ & $2.319 E-01$ \\
4 & $L$ & $1.285 E-05$ & $1.989 E-03$ & $6.062 E-06$ & $8.645 E-04$ & $2.495 E-06$ & $2.060 E-03$ \\
& $U$ & $1.698 E-05$ & $2.298 E-03$ & $1.955 E-06$ & $2.962 E-03$ & $1.285 E-06$ & $2.724 E-03$ \\
5 & $L$ & $1.050 E-07$ & $1.255 E-03$ & $4.649 E-08$ & $9.859 E-04$ & $1.720 E-08$ & $7.284 E-04$ \\
& $U$ & $1.847 E-07$ & $1.159 E-04$ & $2.712 E-08$ & $2.320 E-04$ & $9.465 E-08$ & $4.912 E-04$ \\
6 & $L$ & $7.390 E-10$ & $1.156 E-05$ & $3.058 E-10$ & $9.433 E-07$ & $9.920 E-11$ & $3.428 E-06$ \\
& $U$ & $1.350 E-09$ & $3.118 E-06$ & $7.110 E-11$ & $5.122 E-06$ & $5.014 E-11$ & $5.347 E-06$ \\
\hline
\end{tabular}


Table 10. Error Residual for $\widetilde{u_{2}}(x, \alpha)$ (Problem 2)

\begin{tabular}{cccccccc}
\hline & \multicolumn{4}{c}{$\alpha=0.3$} & \multicolumn{2}{c}{$\alpha=0.6$} & \multicolumn{2}{c}{$\alpha=0.9$} \\
\cline { 1 - 7 }$n$ & $i$ & $\mathrm{ADM}$ & $\mathrm{MT}$ & $\mathrm{ADM}$ & $\mathrm{MT}$ & $\mathrm{ADM}$ & $\mathrm{MT}$ \\
\hline 3 & $L$ & $3.751 E-04$ & $1.147 E-01$ & $2.955 E-04$ & $5.029 E-02$ & $1.979 E-04$ & $5.800 E-03$ \\
& $U$ & $3.081 E-04$ & $4.793 E-02$ & $3.550 E-05$ & $5.832 E-02$ & $1.570 E-04$ & $3.058 E-02$ \\
4 & $L$ & $2.525 E-06$ & $1.333 E-02$ & $2.198 E-06$ & $9.011 E-03$ & $1.211 E-06$ & $5.735 E-03$ \\
& $U$ & $4.310 E-06$ & $1.150 E-03$ & $1.956 E-06$ & $1.755 E-03$ & $9.418 E-06$ & $3.528 E-03$ \\
5 & $L$ & $4.271 E-08$ & $1.581 E-03$ & $1.928 E-08$ & $1.080 E-03$ & $7.010 E-09$ & $6.880 E-04$ \\
& $U$ & $5.304 E-08$ & $2.046 E-05$ & $7.163 E-09$ & $2.097 E-03$ & $4.217 E-09$ & $3.332 E-04$ \\
6 & $L$ & $4.399 E-09$ & $2.319 E-05$ & $1.501 E-10$ & $1.066 E-05$ & $4.090 E-11$ & $3.979 E-06$ \\
& $U$ & $8.205 E-10$ & $2.289 E-07$ & $3.357 E-11$ & $5.404 E-07$ & $1.672 E-11$ & $6.306 E-07$ \\
\hline
\end{tabular}

\section{Conclusions}

The results obtained from the two given problems, have been shown that the Adomian decomposition method and modified technique are powerful and efficient techniques to find the approximate solution for both linear and non-linear fuzzy system of Volterra integro-differential equations of the second kind according to the numerical results, mentioned in the tables and graphs. Therefore, increasing the number of iterations in the Adomian decomposition method and modified technique, make the approximate solution tends to the exact solution. For the non-linear problem, we conclude that the numerical results by using the numerical solution with the Simpson rule close to the exact solution more than the numerical solution with the trapezoidal rule. Finally, we conclude that the methods are extremely quick and simple to converge for solving any equation or system for any value of $\alpha$, and it provides more accurate results and therefore is more advantageous.

\section{Acknowledgements}

The College of Computer Science and Mathematics at the University of Mosul, Iraq, provided funding for this study.

\section{References}

[1] Hermann Brunner. Volterra integral equations: an introduction to theory and applications. Cambridge University Press, Cambridge monographs on applied and computational Mathematics, 2017.

[2] H. J. Zimmermann. Fuzzy set theory and its application. Springer science, 2001.

[3] Abdul-Majid Wazwaz. A new algorithm for calculating Adomian polynomials for nonlinear operators. Applied mathematics and computation, 111(1):53-69, 2000.

[4] George Adomian. Nonlinear Stochastic Operator Equations. Academic Press, New York, 1986. 
[5] George Adomian. Nonlinear Stochastic Systems Theory and Applications to Physics. Kluwer Academic Publishers, Dordrecht, 1989.

[6] George Adomian. Solving Frontier Problems of Physics: The Decomposition Method. Kluwer Academic Publishers, Dordrecht, 1994.

[7] Ahmed A. Hamoud, and Kirtiwant P. Ghadle. Modified Adomian decomposition method for solving fuzzy Volterra-Fredholm integral equation. The Journal of the Indian Mathematical Society, 85(1-2):53-69, 2018.

[8] Ahmed A. Hamoud, Ali Dhurgham Azeez, and Kirtiwant P. Ghadle. A study of some iterative methods for solving fuzzy Volterra-Fredholm integral equations. Indonesian Journal of Electrical Engineering and Computer Science 11(3):1228-1235, 2018.

[9] Ahmed A. Hamoud, and Kirtiwant P. Ghadle. Homotopy analysis method for the first order fuzzy Volterra-Fredholm integro-differential equations. Indonesian Journal of Electrical Engineering and Computer Science, 11(3):857-867, 2018.

[10] M.B. Issa, A. Hamoud, A. Sharif, K. Ghadle, and G. Giniswamy. Modified Adomian decomposition method for solving fuzzy integro-differential equations. Canadian Journal of Applied Mathematics, 3(1):37-45, 2021.

[11] Abdul-Majid Wazwaz. A reliable modification of Adomian decomposition method. Applied Mathematics and Computation, 102(1):77-86, 1999.

[12] Aytac Arikoglu, and Ibrahim Ozkol. Solutions of integral and integro-differential equation systems by using differential transform method. Computers and Mathematics with Applications, 56(9):2411-2417, 2008.

[13] Ahmed A. Hamoud, and Kirtiwant P. Ghadle. Modified Laplace decomposition method for fractional Volterra-Fredholm integro-differential equations. Journal of Mathematical Modeling 6(1):91-104, 2018.

[14] Lamiaa H. Al-Taee, and Waleed Al-Hayani. Solving systems of non-linear Volterra integral equations by combined Sumudu transform-Adomian decomposition method. Iraqi Journal of Science, 62(1):252-268, 2021.

[15] Dalal Adnan Maturi, and Eman Ahmed M. Simbawa. The modified decomposition method for solving Volterra Fredholm integro-differential equations using Maple. International Journal of GEOMATE, 18(67):84-89, 2020.

[16] Hassan Ibrahim, and Peter Vanenchii Ayoo. Approximation of systems of Volterra integro-differential equations using the new iterative method. International Journal of Science and Research, 4(5):332-336, 2015.

[17] M. I. Berenguer, D. Gáme, and A.J. López Linares. Solution of systems of integrodifferential equations using numerical treatment of fixed Point. Journal of Computational and Applied Mathematics, 315(1):343-353, 2017. 
[18] Jafar Biazar, and Hossein Aminikhah. A new technique for solving nonlinear integraldifferential equations. Computers and Mathematics with Applications, 58:2084-2090, 2009 .

[19] Mohammed S. Bani Issaa, Ahmed A. Hamoud, and Kirtiwant P. Ghadle. Numerical solutions of fuzzy integro-differential equations of the second kind. Journal of Mathematics and Computer Science, 23(2):67-74, 2020.

[20] R. Mastani Shabestari, R. Ezzati, and T. Allahviranloo. Solving fuzzy Volterra Integro-differential equations of fractional order by Bernoulli Wavelet method. $A d$ vances in Fuzzy Systems, 2018:1-11, 2018.

[21] Manmohan Das, and Dhanjit Talukdar. Method for solving fuzzy integro-differential equation by using fuzzy Laplace transformation. International Journal of Scientific E Technology Research, 3(5):291-295, 2014.

[22] Nasser Mikaeilvand, Sakineh Khakrangin, and Tofigh Allahviranloo. Solving fuzzy Volterra integro-differentiale quation by fuzzy differential transform method. Proceedings of the 7th conference of the European Society for Fuzzy Logic and Technology (EUSFLAT-11), 2011.

[23] A. J. Abdulqader. Numerical Solution for Solving System of Fuzzy Nonlinear Integral Equation by Using Modified Decomposition Method. Journal of Mathematics Research, 10 (1): 32-43, 2018.

[24] Mahdy Baghmisheh, and Reza Ezzati. Numerical solution of nonlinear fuzzy Fredholm integral equations of the second kind using hybrid of block-pulse functions and Taylor series. Advances in Difference Equations, 2015(1):1-15, 2015.

[25] Jihan Hamaydi, and Naji Qatanani. Computational Methods for Solving Linear Fuzzy Volterra Integral Equation. Journal of Applied Mathematics, 2017:1-12, 2017.

[26] A. Molabahrami, A. Shidfar, and A. Ghyasi. An analytical method for solving linear Fredholm fuzzy integral equations of the second kind. Computers and Mathematics with Applications, 61(9):2754-2761, 2011.

[27] M. R. Nourizadeh, N. Mikaeilvand, and T. Allahviranloo. Existence and uniqueness solutions of fuzzy integration-differential mathematical problem by using the concept of generalized differentiability. AIMS Mathematics, 4(5):1430-1449, 2019.

[28] Nasser Mikaeilvand, Zahra Noeiaghdam, Samad Noeiaghdam, and Juan J. Nieto. A novel technique to solve the fuzzy system of equations, Mathematics, 8(5):850, 2020.

[29] Atiyeh Mashhadi Gholam, and Reza Ezzati. Solving linear fuzzy Fredholm integral equations of the second kind via iterative method and Simpson Quadrature Rule: A review. TWMS Journal of Pure and Applied Mathematics, 8(2):121-147, 2017. 
[30] Abdul-Majid Wazwaz. Linear and nonlinear integral equations methods and applications. Springer, higher education Prees, 2011.

[31] Hsien-Chung Wu. The fuzzy Riemann integral and its numerical integration. Fuzzy Sets and Systems, 110(1):1-25, 2000. 